\title{
Histologic Localization of PLAG1 (Pleomorphic Adenoma Gene 1) in Pleomorphic Adenoma of the Salivary Gland: Cytogenetic Evidence of Common Origin of Phenotypically Diverse Cells
}

\author{
Maria Debiec-Rychter, Isabelle Van Valckenborgh, Christel Van den Broeck, \\ Anne Hagemeijer, Wim J. M. Van de Ven, Koen Kas, Boudewijn Van Damme, and \\ Marianne L. Voz \\ Laboratory for Cytogenetics and Molecular Genetics of Human Malignancies (MD-R, AH), Center for Human \\ Genetics, Katholieke Universiteit Leuven, Leuven; Laboratory for Molecular Oncology (IVV, WJMV, KK, MLV), \\ Center for Human Genetics, Katholieke Universiteit Leuven and Flanders Interuniversity Insitute for Biotechnology, \\ Leuven; Department of Pathology (CVB, BVD), Katholieke Universiteit Leuven, Leuven; and Department of \\ Otorhinolaryngology (IVV), Head and Neck Surgery, Katholieke Universiteit Leuven, Leuven, Belgium
}

\begin{abstract}
SUMMARY: Pleomorphic adenoma gene 1 (PLAG1), a zinc finger transcription factor gene, is consistently rearranged and overexpressed in human pleomorphic adenomas of the salivary glands with $8 q 12$ translocations. In this report, we describe the immunohistochemical localization of PLAG1 protein in pleomorphic adenomas of the salivary gland and corresponding normal tissue, in relation to cytokeratin, vimentin, and BCL-2 expression. Normal salivary gland tissue was not immunoreactive for PLAG1. In primary pleomorphic adenomas, cells strongly immunoreactive for PLAG1 were detected in the outer layer of tubulo-ductal structures, which are thought to be the origin of cells with bi-directional, epithelial, and mesenchymal phenotypes. In contrast, epithelial cells with abundant cytokeratin in the inner tubulo-ductal structures only sporadically expressed PLAG1. BCL-2 immunoreactivity was found mainly in the cells surrounding the tubulo-ductal structures and in the solid undifferentiated cellular masses, within the areas that had moderate PLAG1 immunoreactivity. The variability of PLAG1 expression in neoplastic cells seemed to reflect the morphologic heterogeneity that correlated with the stage of differentiation of the tumor cells. Immunohistochemical/cytogenetic evaluation of two pleomorphic adenomas with $t(3 ; 8)(p 21 ; q 12)$ or $t(5 ; 8)(p 13 ; q 12)$ translocations demonstrated the clonal nature of immunophenotypically diverse cells. This finding confirms the theory that pleomorphic adenoma cells share a common single-cell origin, most likely from the epithelial progenitor basal duct cells. (Lab Invest 2001, 81:1289-1297).
\end{abstract}

leomorphic adenomas, the most common benign
tumors of the salivary gland, are classified as
tumors of epithelial origin (Seifert et al, 1990). They are
morphologically characterized by a biphasic pattern
containing both epithelial and mesenchymal areas
(Dardick et al, 1982, 1987; Erlandson et al, 1984;
Palmer et al, 1985). The epithelial component of these
tumors contains double-layered cystic, tubular, or
solid structures, usually with a distinct inner layer

Received June 6, 2001

$M D$-R and IVV contributed equally to this report. This work was supported by the Geconcerteerde Onderzoekacties 1997-2001, the Fonds voor Wetenschappelijk Onderzoek Vlaanderen (FWO), and the Belgian program on Interuniversity Poles of Attraction initiated by the Belgian State, Prime Minister's Office, Science Policy Programming. MLV is a Chercheur Qualifie from the Fonds National de la Recherche Scientifique. IVV is a doctoral fellow of the FWO.

Address reprint requests to: Dr. Maria Debiec-Rychter, Center for Human Genetics, Katholieke Universiteit Leuven, Herestraat 49, B-3000 Leuven, Belgium.E-mail:maria.debiec-rychter@med.kuleuven.ac.be facing a lumen and an outer layer facing the stromal components. In some of the epithelial clusters, these layers expand and lead to masses of spindled cells in connection with the tumoral stroma. Aberrantly differentiated cells in the outer layers of tubulo-ductal structures (so-called "modified myoepithelial" or myoepithelium-like cells) have a bi-directional, epithelial and mesenchymal phenotype (Palmer et al, 1985; Sunardhi-Widyaputra and Van Damme, 1995; van den Oord et al, 1993). These cells are thought to play an important role in the formation of the extracellular matrix and thereby in the creation of the histologic diversity that is characteristic of pleomorphic adenomas (Neureiter et al, 1999). Although these cells comprise a major component of pleomorphic adenomas and are responsible for the spectrum of growth patterns seen in these tumors, the cytologic origin of the chromosomal abnormalities in pleomorphic adenoma has not been clearly established.

The pleomorphic adenoma gene 1 (PLAG1) on chromosome $8 \mathrm{q} 12$ plays a significant role in the develop- 
ment of pleomorphic adenoma of the salivary gland. Activation of PLAG1 gene expression is the result of recurrent chromosomal translocations that may involve a large number of different partners (Bullerdiek et al, 1987). In the two most frequent translocations, $\mathrm{t}(3 ; 8)(\mathrm{p} 21 ; \mathrm{q} 12)$ and $\mathrm{t}(5 ; 8)(\mathrm{p} 13 ; \mathrm{q} 12)$, the replacement of the PLAG1 promoter, which is inactive in mature salivary glands, by a strong promoter derived from the $\beta$-catenin (CTNNB1) and leukemia inhibitory factor receptor (LIFR) genes, respectively, leads to abnormal expression of PLAG1 in the tumor cells (Kas et al, 1997; Voz et al, 1998). Because PLAG1 seems to act as a transcriptional regulator, this ectopic expression probably results in deregulation of target genes (Kas et al, 1998; Voz et al, 2000).

In this report, we describe the histologic localization of the PLAG1 protein in salivary gland tumors with $8 \mathrm{q} 12$ translocations. Immunohistochemistry was performed on pleomorphic adenomas using antibodies raised against the $\mathrm{C}$ - or $\mathrm{N}$-terminal parts of the PLAG1 protein. PLAG1 immunoreactivity was related to the protein expression of cytokeratins and vimentin, histologic markers of epithelial and mesenchymal differentiation, respectively, and BCL-2, a marker of cellular immaturity. To determine the histogenetic origin of the cells with chromosome translocations, a combined immunocytologic/cytogenetic approach was developed that enabled simultaneous ascertainment of cytogenetic aberrations and immunophenotypic features in individual cells.

\section{Results}

\section{Generation of Specific PLAG1 Antibodies}

GST-PLAG1 (N2-C41) and GST-PLAG1 (N341-C500) fusion proteins were expressed in Escherichia coli and the fusion proteins were purified on Glutathione Sepharose columns and injected into rabbits to obtain the PEM195 and PEM190 antibodies, respectively (see "Material and Methods"). The affinity and specificity of the antibodies were tested by Western blot analysis. For that purpose, we expressed PLAG1 by in vitro transcription and translation of the PCDNA3PLAG1 expression vector that contains the complete open reading frame of PLAG1 preceded by its own Kozak consensus translation start site (Voz et al, 2000). This expression leads to the generation of three isoforms, the full-length PLAG1 protein and two alternative isoforms generated by the initiation of the translation at methionines 83 and 100. As seen in Figure 1, the PEM190 antibody, directed against the C-terminal part of PLAG1, recognized the three isoforms (Lane 1). In contrast, the PEM195 antibody, directed against the N-terminal part of PLAG1, recognized only the full-length protein (Lane 3). Preimmune sera did not detect any PLAG1 proteins (Lanes 2 and 4).

\section{Histologic and Immunohistochemical Study}

PLAG1 Immunoreactivity in Pleomorphic Adenomas versus Normal Salivary Glands. PLAG1 expression

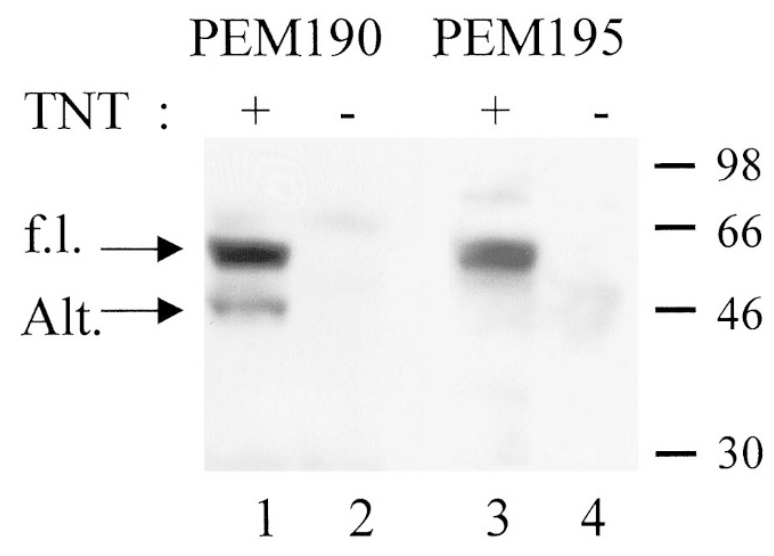

Figure 1.

Western blot analysis. Five microliters of in vitro transcription and translation of pCDNA3-PLAG1 (Lanes 1 and 3) and of mock translated products (Lanes 2 and 4) were subjected to SDS-PAGE, transferred to nitrocellulose, and incubated with PEM190 (Lanes 1 and 2) and with PEM195 antibodies (Lanes 3 and 4). The arrow marked f.I. indicates the full length PLAG1 protein. The arrow marked Alt. shows the two alternative isoforms generated by the initiation at met 83 and met 100, which co-migrated in a single band.

was not detected in normal salivary gland tissue (Fig. 2, E and F), in agreement with the absence of PLAG1 transcripts in this tissue (Kas et al, 1997; Voz et al, 1998). In contrast, nuclear immunoreactivity was found in up to $75 \%$ to $80 \%$ of the various cells in the pleomorphic adenoma with a $t(3 ; 8)(p 21 ; q 12)$ translocation that has been shown to express high level of PLAG1 transcripts (Kas et al, 1997). Immunoreactivity was mainly restricted to the nuclei and was stronger with PEM195 (Fig. 2D) than PEM190 (Fig. 2C). Weak PLAG1 immunoreactivity was found in the inner cell layer of tubulo-ductal structures, and strong immunoreactivity was found in the outer cell layer (Fig. 2, C and D). Moderate PLAG1 immunoreactivity was also found in the solid undifferentiated cellular areas. As expected, no immunoreactivity was observed with the preimmune sera (Fig. 2, A and B).

PLAG1 Immunoreactivity versus Cytokeratin, Vimentin, and $\mathrm{BCl}-2$ Expression. To reveal which types of cell expressed PLAG1, serial sections were cut and processed for immunohistochemistry with different antibodies. In the nontumoral parts of the salivary glands, cytokeratin expression was restricted to acinar cells and ducts (data not shown). In pleomorphic adenoma, strong cytokeratin immunoreactivity was found in the inner layer of tubulo-ductal structures, whereas the outer layer was less immunoreactive. Scattered neoplastic cells in the undifferentiated cellular mass were also immunoreactive (Fig. 3D).

In normal parotid tissue, vimentin expression was found only in stromal areas of the gland (not shown). In salivary gland pleomorphic adenomas, vimentin immunoreactivity was found in the undifferentiated cellular areas and in scattered cells that surround the epithelial gland-like structures (Fig. 3E). Some of the scattered cells surrounding the epithelial gland-like structures were also immunoreactive for cytokeratins.

In normal salivary glands, BCL-2 expression was restricted to the basal and dark cells of excretory and 



Figure 2.

Using the preimmune serum of PEM190 (A) and PEM195 (B), there was no immunoreactivity in the primary pleomorphic adenoma sections. Moderate (C) and strong (D) immunoreactivity for pleomorphic adenoma gene 1 (PLAG1) was found in the nuclei of the outer layer $(0)$ of the tubulo-ductal structures and in the undifferentiated cellular masses $(m)$ using PEM190 (C) and PEM195 (D) antibodies, respectively. Immunoreactivity to PLAG1 using PEM190 (E) and PEM195 (F) antibodies was not found in the normal salivary gland. Cryostat sections, PAP with Hemalum counterstain. Original magnification, $\times 250$.

striated ducts, and to some stromal inflammatory cells (not shown). In pleomorphic adenomas, cells surrounding tubulo-ductal structures expressed BCL-2 (Fig. 3C). Strong immunoreactivity for BCL-2 was also found in solid undifferentiated cellular masses. Some of the solid undifferentiated cellular masses were also immunoreactive for PLAG1. Overall, however, the strongest PLAG1 immunoreactivity was found mainly in the outer layer of tubulo-ductal structures. In contrast, stromal and undifferentiated tumoral masses with extensive BCL-2 and vimentin expression had moderate PLAG1 immunoreactivity (Fig. 3, A and B).

The distribution of PLAG1, cytokeratin, and vimentin proteins in normal human salivary gland and pleomorphic adenoma is summarized in Table 1. All controls 


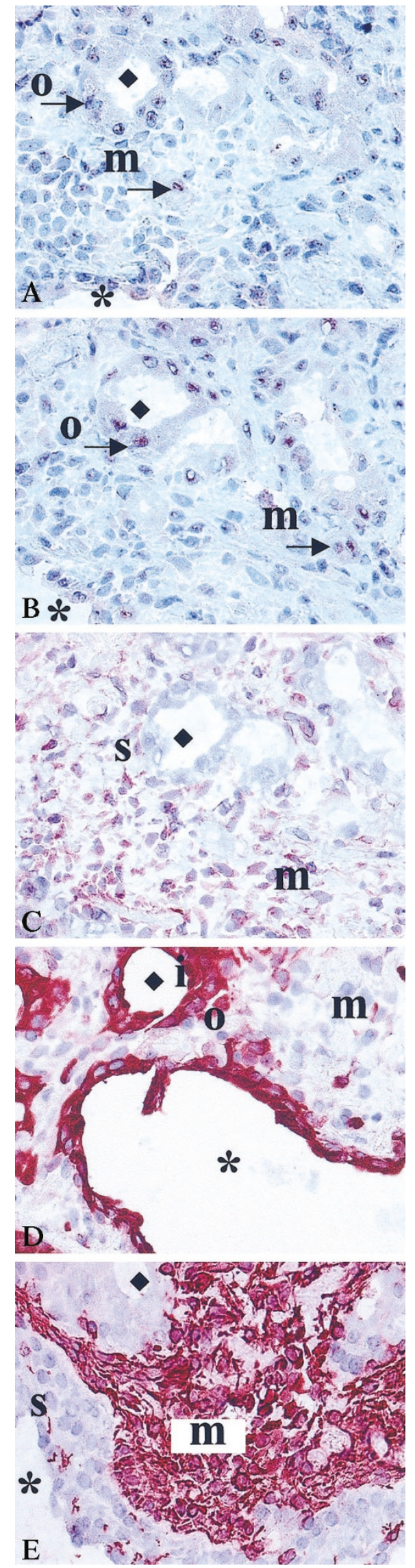

(replacing the primary antibody with nonimmune rabbit or mouse serum) were negative.

\section{Molecular Cytogenetic Analysis}

On metaphase cells of the pleomorphic adenoma with the $46, \mathrm{XY}, \mathrm{t}(5 ; 8)(\mathrm{p} 13 ; \mathrm{q} 12)$ karyotype, a YAC 906F4/ LIFR-specific probe revealed a green signal at band $5 \mathrm{p} 13$ of the normal chromosome 5 and a split signal at the breakpoint sites of both derivatives chromosomes der(5) and der(8). A YAC 935E9/PLAG1-specific probe revealed red signals at band $\mathrm{q} 12$ on the normal chromosome 8 and a fusion with the signal from the YAC 906F4 probe on chromosome der(8), confirming the involvement of PLAG1 and LIFR genes in the 5;8 translocation (not shown). Interphase fluorescence in situ hybridization (FISH) analysis, using these probes, revealed two green signals, one red signal, and one fusion (yellow) signal in $90 \%$ of the nuclei (Fig. 4, B and D).

On metaphase cells of the pleomorphic adenoma with the $46, X Y, t(3 ; 8)(p 21 ; q 12)$ karyotype, a YAC 750D3/CTNNB1-specific probe revealed a green signal at band 3p21 of the normal chromosome 3 and a split signal at the breakpoint sites of both derivatives chromosomes der(3) and der(8). A YAC 935E9/ PLAG1-specific probe revealed red signals at band q12 on the normal chromosome 8 and a fusion with the signal from the YAC 750D3 probe on chromosome $\operatorname{der}(8)$, confirming the involvement of PLAG1 and CTNNB1 genes in the 3;8 translocation. Interphase FISH analysis revealed two green signals, one red signal, and one fusion (yellow) signal in approximately $80 \%$ of the nuclei (not shown).

\section{Triple-color Immunofluorescence Cytochemistry and FISH Analysis on Pleomorphic Adenomas Propagated In Vitro}

Using both the PEM190 and PEM195 antibodies, clear immunoreactivity was found in up to $90 \%$ of the cells from the tumors carrying specific $t(3 ; 8)$ and $t(5 ; 8)$ translocations. PLAG1 immunoreactivity was present mainly in the nuclei, whereas the cytoplasm was immunoreactive for cytokeratins and vimentin. A large degree of heterogeneity in cellular size and shape and intensity of PLAG1 immunoreactivity was observed (Fig. 4, A and C). In contrast, only weak nuclear anti-PLAG1 immunoreactivity was found in approximately $10 \%$ of the control pleomorphic adenoma cells

\section{Figure 3.}

Serial sections of primary pleomorphic adenoma. Immunoreactivity to PLAG1 using PEM190 (A) and more intense immunoreactivity to PEM195 (B) antibodies was found in the nuclei of the outer layer of the tubulo-ductal structures $(0)$ and in the undifferentiated cell clusters $(m)$. Immunoreactivity for BCL-2 (C) was found in cells surrounding $(s)$ the tubulo-ductal structures and in the undifferentiated cellular mass $(m)$. Cells strongly immunoreactive for cytokeratin (D) were found in the inner $(i)$ layer of the tubulo-ductal $(\checkmark)$ and cystic structures $\left({ }^{*}\right)$. The outer layer $(0)$ and some cells within the undifferentiated tumoral masses $(m)$ were also immunoreactive, but with markedly less intensity. Strong vimentin immunoreactivity $(E)$ was commonly present in the undifferentiated tumoral mass $(m)$ and in some cells surrounding $(s)$ the cyst structures. Cryostat sections, PAP with Hemalum counterstain. Original magnification, $\times 250$. 
Table 1. Distribution of PLAG1, Cytokeratin, Vimentin and BCL-2 Proteins in Normal Salivary Gland and Pleomorphic Adenomas

\begin{tabular}{|c|c|c|c|c|}
\hline \multirow[b]{2}{*}{ Histology } & \multicolumn{4}{|c|}{ Antigens } \\
\hline & PLAG1 & Cytokeratins & Vimentin & BCL-2 \\
\hline \multicolumn{5}{|l|}{ Normal salivary gland } \\
\hline Acinar cells of glandular structures & - & +++ & - & - \\
\hline \multicolumn{5}{|l|}{ Ductal structures } \\
\hline Light cells & - & +++ & - & - \\
\hline Dark and basal cells & - & +++ & - & + \\
\hline \multicolumn{5}{|l|}{ Pleomorphic adenoma } \\
\hline \multicolumn{5}{|l|}{ Tubulo-ductal structures } \\
\hline Inner rim & $+/-$ & +++ & - & - \\
\hline Outer rim & +++ & + & + & - \\
\hline \multicolumn{5}{|l|}{ Cyst-like structures: } \\
\hline Inner rim & $+/-$ & +++ & - & - \\
\hline Outer rim & +++ & + & + & - \\
\hline Undifferentiated tumoral masses & + & + & +++ & +++ \\
\hline
\end{tabular}

with $\mathrm{t}(3 ; 12)$ or $\mathrm{t}(\mathrm{X} ; 2)$ aberrations, confirming the specificity of the antibodies and ruling out the possibility of PLAG1 induction during in vitro propagation.

After 3 days of culturing, round, epithelioid cells, with big nuclei and a narrow rim of cytoplasm represented approximately one third of the cellular populations. The majority of the epithelioid cells were clearly immunoreactive for cytokeratin, weakly immunoreactive for vimentin, and moderately to strongly immunoreactive to both anti-PLAG1 antibodies (Fig. 4, A and C, arrowheads). Approximately $15 \%$ to $20 \%$ of the epithelioid cells were cytokeratin and PLAG1 but not vimentin immunoreactive (Fig. 4C, arrow). Expression of PLAG1 protein in these cells was much lower than in the cells with bi-directional, epithelial and mesenchymal phenotype, as assessed by the immunofluorescence intensity. Few epithelioid cells were immunoreactive for cytokeratin but not for vimentin or PLAG1.

Elongated or polygonal, fibroblast-like cells that frequently formed filopodia and long cytoplasmic extensions with bulbous endings represented the other cellular compartment proliferating in vitro. The preponderance of fibroblast-like cells $(80 \%)$ were also immunoreactive to anti-vimentin and anti-cytokeratin. However, the cytokeratin immunoreactivity was less intense than the vimentin. In the majority of these cells, immunoreactivity was evident with both antibodies directed against PLAG1, although not as intense as in the epithelioid cells. In the remaining $20 \%$ of the fibroblast-like cells the expression of vimentin and PLAG1 (indicated by the arrow on Fig. 4A) but not cytokeratin was observed. Again, immunoreactivity to PLAG1 in these cells was weaker, than in the cells with bi-directional phenotype, as assessed by immunofluorescence intensity.

All cells with PLAG1 immunoreactivity carried chromosomal $3 ; 8$ or $5 ; 8$ translocations (Fig. 4, B and D). A small fraction of cells, however, had cytokeratin or vimentin immunoreactivity and carried the translocations but did not express PLAG1 by immunofluores- cence (not shown). The presence of the translocation in cells of epithelial, mesenchymal, or bidirectional phenotype unambiguously demonstrated the clonal nature of the different components of the tumor (Fig. 4, A, Aa, Ab, Ac; C, Ca, Cb, Cc).

\section{Discussion}

In this report, we described the histologic pattern of PLAG1 expression in pleomorphic adenomas of the salivary gland. We used two polyclonal antibodies obtained by immunizing rabbits with two nonoverlapping parts of the PLAG1 protein (the amino acids 2 to 41 for PEM195 and 341 to 500 for PEM190). Several lines of evidence indicate that these antibodies react specifically with PLAG1 in immunohistochemistry and immunofluorescence studies. Firstly, these two completely different antibodies gave exactly the same expression pattern on pleomorphic adenomas whereas no immunoreactivity was observed with the preimmune sera. Secondly, no immunoreactivity was observed with these two antibodies in normal salivary gland, in which no PLAG1 transcripts were detected by Northern blot analysis in a previous study (Kas et al, 1997). In contrast, strong reactivity was observed on pleomorphic adenomas carrying $3 ; 8$ or $5 ; 8$ translocations that had shown high level of PLAG1 transcripts. Finally, pleomorphic adenomas without PLAG1 gene rearrangements did not show any PLAG1 reactivity by immunofluorescence.

PLAG1 expression was restricted to the neoplastic component but widespread within the tumoral tissue. Although the inner layer of tubulo-ductal and cyst-like structures showed no or only occasional PLAG1 immunoreactivity, the outer layer and cells that expand from the outer layer toward tumoral stroma were strongly immunoreactive. In the dense undifferentiated solid masses, most cells were moderately immunoreactive, but few cells were intensely immunoreactive. This pattern of PLAG1 expression seemed to correlate with the lack of differentiation of the tumoral 

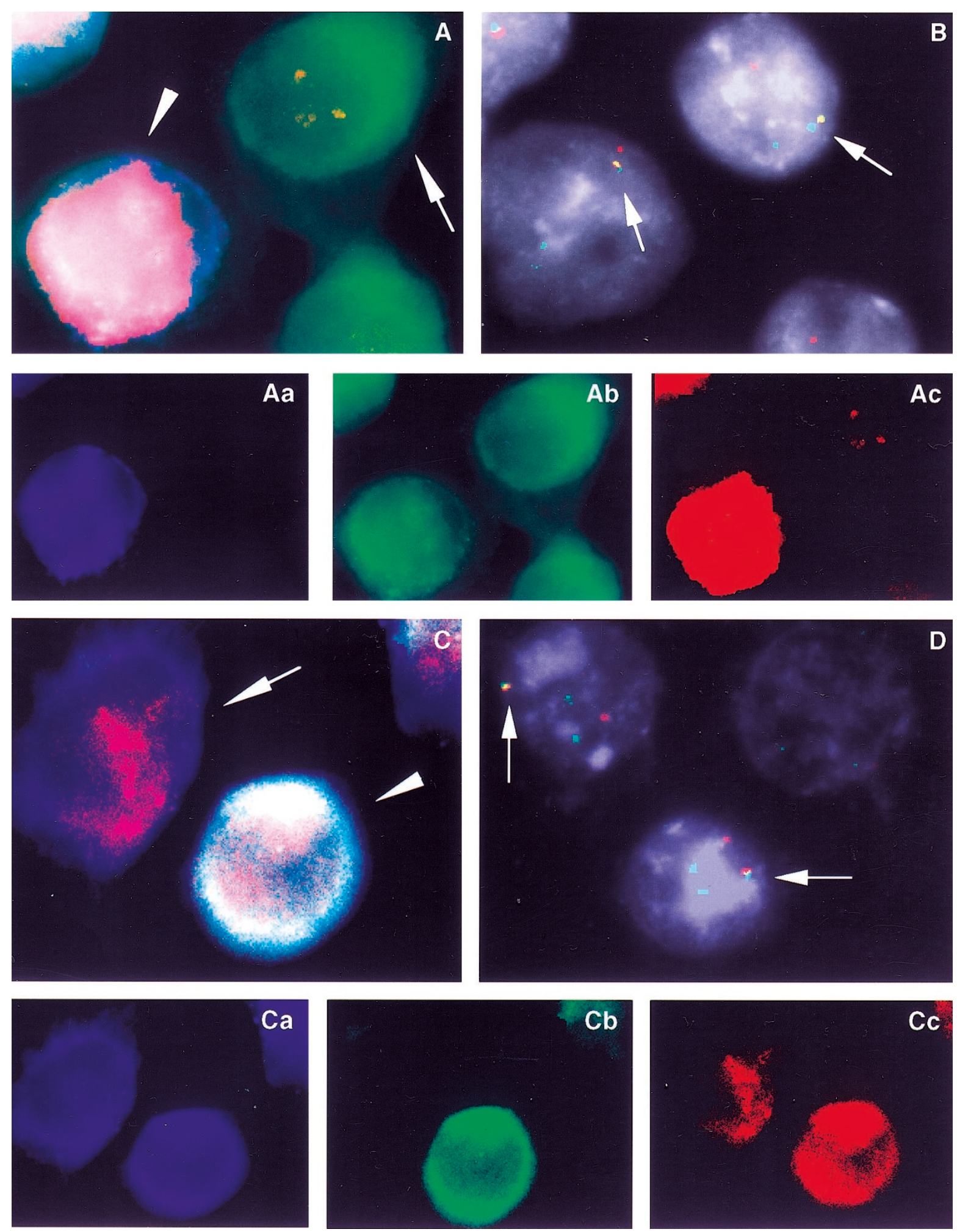

\section{Figure 4.}

Triple-color, immunofluorescence images of PLAG1, cytokeratin, and vimentin expression in human pleomorphic adenoma cells carrying $t(5 ; 8)(p 13 ; q 12)$ translocation and propagated for 3 days in vitro (A and $C$ ). Anti-PLAG1, anti-vimentin, and anti-cytokeratin immunoreactivity was revealed using Texas-Red-conjugated donkey anti-rabbit (red), FITC-conjugated rabbit anti-goat (green), and AMCA-conjugated anti-mouse (blue) secondary antibodies, respectively. Individual color images corresponding to superimposed images in $\mathrm{A}$ and $\mathrm{C}$ are given in a, b, and $\mathrm{C}$. Long arrowheads indicate bi-directionally differentiated cells with high PLAG1 expression (A and C); arrows indicate cells with either vimentin (A) or cytokeratin (C) immunoreactivity that had low levels of PLAG1 expression. Double-color interphase fluorescence in situ hybridization (FISH) analysis of cells corresponding to A and C are given in B and D, respectively. FISH was performed using a combination of digoxigenin-labeled YAC 935E9 (red signals) and biotin-labeled YAC906F4 (green signals), which map to the PLAG1/8q12 and LIFR/5P13 genes, respectively, clones as molecular probes. The presence of translocation is visualized by the juxtaposed, overlapping green and red signals, indicated by arrows. 
cells. Indeed, the areas that were immunoreactive for BCL-2 were also immunoreactive for PLAG1. BCL-2 protects cells from apoptosis and its expression is seen in undifferentiated cells in association with cellular immaturity (Hockenbery et al, 1993). Moreover, some cells within the outer rim, cells surrounding tubulo-ductal structures, and cells in solid tumoral masses expressed vimentin; a reflection of their aberrant differentiation during the neoplastic transformation. However, the most differentiated epithelial cells of the inner tubulo-ductal structures, which show normal cytokeratin presence, only sporadically expressed PLAG1. Our findings indicate that the inner layer cells follow a normal rather than an aberrant differentiation, whereas there is an abnormal differentiation in the outer layer of tubulo-ductal structures of pleomorphic adenomas.

Our immunohistochemical findings were further confirmed by the immunofluorescence cytochemical studies performed on cultured cells. Both cases with specific $3 ; 8$ and $5 ; 8$ translocations associated with PLAG1 rearrangements revealed clear widespread PLAG1 expression, whereas the majority of pleomorphic adenoma cells without $8 \mathrm{q} 12 /$ PLAG1 rearrangements did not have PLAG1 immunoreactivity. In cases with recurrent $3 ; 8$ and $5 ; 8$ translocations, the majority of the cells showed bi-directional, anti-cytokeratin and anti-vimentin immunoreactivity, with the expression of PLAG1 in these cells being most prominent. Additionally, we identified minor fractions of cells that had PLAG1 immunoreactivity but selective cytokeratin or vimentin immunoreactivity. Judging from the immunoreactivity intensity, the expression of PLAG1 in these cells was moderate or low. Irrespective of their phenotypes, however, all cells that expressed PLAG1 protein carried specific chromosomal translocations. These results confirm the role of specific translocations in PLAG1 up-regulation, unambiguously demonstrate the monoclonal origin of pleomorphic adenomas, and provide evidence for a common single cell origin for the epithelial and the mesenchymal component of these tumors. They confirm the conclusion of clonality studies performed by others using the human androgen receptor gene (HUMARA) assay (Lee et al, 2000; Noguchi et al, 1996). Our results and the results of others could indicate that pleomorphic adenoma cells share a common origin from a single uncommitted cell that possesses the capacity for differentiation into tumor cells of epithelial, mesenchymal, or both phenotypes. Alternatively, pleomorphic adenomas could develop from the committed progenitor cells, eg, from the epithelial basal ductal cells (Batsakis, 1980; Eversole, 1971; Sunardhi-Widyaputra and Van Damme, 1995). Because of neoplastic transformation, these progenitor cells could acquire the ability for uncontrolled proliferation and for conversion into mesenchymal cells. The mechanisms by which cells activate putative mesenchymal master genes and turn off epithelial genes, are only partially known (Hay, 1995). In this context, the biologic implications of abnormal PLAG1 expression in pleomorphic adenoma cells are not yet fully understood. More data on genes interact- ing with or induced by overexpressed PLAG1 need to be accumulated before any conclusive interpretation of pleomorphic adenoma tumorigenesis is possible.

\section{Materials and Methods}

\section{Construction, Production, and Purification of Recombinant GST-PLAG1 Fusion Proteins and Generation of Specific PLAG1 Antibodies}

The amino terminal part of PLAG1 fused to GST, GST-PLAG1 (N2-C41), was obtained by digesting GST-PLAG1 (N2-C244) (Voz et al, 2000) with Stul and Xhol, blunt ending, and recircularization. The carboxyl terminal part of PLAG1 fused to GST, GST-PLAG1 (N341-C500), was obtained by digesting GST-PLAG1 (N236-C500) with EcoRl and Ndel, blunt ending, and recircularization. The GST-PLAG1 (N236-C500) was obtained by cloning an EcoRI-Xhol-digested PCRamplified fragment into the EcoRI-Xhol-digested pGSTag vector (Ron and Dressler, 1992). The oligonucleotides used to generate the constructs were G8N236 (5'-CCCGAATTCACAATCAAGAGCTTCTGAAG-3'), and G8C500 (5'-GGGCTCGAGCTACTGAAAAGCTTGATGGAAAC-3'). GST-PLAG1 (N2-C41) and GST-PLAG1 (N341-C500) were expressed in Escherichia coli BL-21 cells and purified on Glutathione Sepharose 4B beads (Pharmacia, Piscataway, New Jersey) according to the manufacturer's protocol. The protein sizes were estimated by sodium dodecyl sulfate-polyacrylamide gel electrophoresis (SDS-PAGE) followed by Coomassie blue staining, and concentrations were determined by comparison with a well defined concentration marker. These purified proteins were used for the antibody generation. Rabbits were injected with the purified proteins (300 $\mu \mathrm{g}$ to $700 \mu \mathrm{g}$ of protein for each injection) in complete adjuvant, and boosting with proteins diluted in incomplete adjuvant were repeated every 4 weeks for 20 weeks. The rabbit serum was tested for immunoreactivity against GST-PLAG1 and full-length PLAG1 proteins expressed in reticulocytes lysates by Western blot analysis.

\section{Western Blot Analysis}

PLAG1 proteins were obtained by in vitro translation (TnT Kit; Promega, Madison, Wisconsin) using the pCDNA3-PLAG1 expression vector containing the complete open reading frame of PLAG1 preceded by its own Kozak sequence (Voz et al, 2000). Five microliters of the translated products were loaded on a $10 \%$ polyacrylamide gel. After separation by size, proteins were transferred by electrophoresis to nitrocellulose membranes. The membranes were incubated with the antibodies against the amino terminal part (PEM195) and the carboxyl terminal part (PEM190) of PLAG1 (diluted 1:1000), followed by an incubation in peroxidase-labeled swine anti-rabbit secondary antibody (diluted 1:2000, PROSAN; DAKO, Glostrup, Denmark). Detection was performed using the Renaissance detection kit (PerkinElmer Life Science Products, Boston, Massachusetts) according to the supplier's instructions. 


\section{Histologic and Immunohistochemical Study}

Serial, $5-\mu m$-thick frozen sections from the nontumoral part of parotid tissue and from primary parotid pleomorphic adenoma with 46,XY,t(3;8)(p21;q12) karyotype were used. Routine hematoxylin and eosin staining confirmed the histopathologic diagnosis. Immunohistochemical studies were completed using polyclonal rabbit antibodies to PLAG1 (diluted 1:60, PEM190 and PEM195) and BCL-2 (diluted 1:10; Dakopatts, Glostrup, Denmark), and monoclonal antibodies against a mixture of low molecular weight cytokeratins (diluted 1:50, KL1, M42128M; Biodesign International, Kennebunk, Maine) and vimentin (diluted 1:30; DAKO), using an indirect peroxidase procedure. Briefly, the serial frozen sections were dried overnight and fixed either in cold, buffered $4.0 \%$ paraformaldehyde (for PLAG1 immunoreactivity) or absolute acetone (for other proteins) for 10 minutes. After fixation, tissue sections were pretreated with either swine or mouse serum for 7 minutes and incubated with the selected primary antibodies. Peroxidase-conjugated swine anti-rabbit (diluted 1:100, SWAR/PO; DAKO) or rabbit anti-mouse Ig (diluted 1:50, RAM/PO; DAKO) were applied as the secondary antibodies, in PBS $(\mathrm{pH}$ 7.2), containing $10 \%$ normal human $A B$-serum. Each antibody incubation was for 30 minutes at room temperature and was followed by three washes in PBS (pH 7.2). Finally, sections were incubated for $10 \mathrm{~min}-$ utes in $0.05 \mathrm{M}$ acetate buffer $(\mathrm{pH} 4.9)$ containing $0.05 \% 3$-amino-9-ethylcarbozole and $0.01 \% \mathrm{H}_{2} \mathrm{O}_{2}$, resulting in a red precipitate, and were lightly counterstained with Mayer's Haemalum stain. Negative controls were prepared by replacing the primary antibody with preimmune serum.

\section{Cytogenetic Analysis}

Four pleomorphic adenomas of the salivary glands were retrieved from the tumor bank of the Center for Human Genetics, Catholic University of Leuven. All were primary tumors originating from the parotid gland in previously untreated patients. Chromosome metaphases of tumor cells were prepared from short-term primary cultures according to a routine method. Two pleomorphic adenomas demonstrated $8 \mathrm{q} 12$ rearrangement, carrying recurrent $t(3 ; 8)(p 21 ; q 12)$ and $t(5 ; 8)(p 13 ; q 12)$ translocations. Two other tumors, with clonal $t(3 ; 12)(p 25 ; q 15)$ and $t(X ; 2)(p 11 ; q 35)$ translocations as the sole anomalies, were selected for the control study.

\section{FISH Analysis}

FISH analysis on metaphase chromosomes was performed to confirm the involvement of CTNNB1 and LIFR genes in cells carrying specific translocations. Double-color FISH analysis, using as probes digoxigenin- or biotin-labeled inter-Alu PCR products of YACs 935E9, 906F4, and 750D3 clones (specific for PLAG1, LIFR, and CTNNB1 genes, respectively) (Kas et al, 1997; Voz et al, 2000), was performed as previously described (Dierlamm et al, 1996). After dehydration and air drying, chromosomes were counterstained with DAPI and mounted with antifade solution. Hybridization signals were analyzed with an Axioplan 2 fluorescence microscope equipped with a cooled CCD camera and run by Cytogen TM system for capture and software analysis (Imstar S.A., Paris, France).

\section{Triple-Color Immunofluorescence and FISH Analysis}

Primary in-situ cultures were obtained from the original single-cell suspensions of tumor cells and grown to $40 \%$ to $60 \%$ confluence for 3 days. The cells were fixed in cold acetone followed by methanol ( 5 minutes each) and air-dried. Cells were permeabilized and blocked in PBS containing $0.2 \%$ Triton X-100 and $0.5 \%$ blocking reagent (Boehringer Mannheim, Mannheim, Germany) for 30 minutes at room temperature. Subsequently, the slides were incubated with polyclonal rabbit antibodies to PLAG1 (diluted 1:50, PEM195 or PEM190) in PBS with $0.5 \%$ blocking reagent $(\mathrm{PB})$ for 1 hour at room temperature; followed by incubation with Texas-Red-conjugated donkey anti-rabbit secondary antibody (diluted 1:100; Amersham Pharmacia Biotech, Uppsala, Sweden) in PB for 1 hour at room temperature. The slides were incubated with the polyclonal goat antibody to vimentin (diluted 1:50, C-20; Santa Cruz Biotechnology, Santa Cruz, California) in PB for 1 hour at room temperature, followed by FITC-conjugated rabbit antigoat secondary antibody (diluted 1:100; Jackson ImmunoResearch Laboratories, West Grove, Pennsylvania) in PB for 1 hour at room temperature. Finally, slides were treated with a monoclonal mouse antibody to cytokeratin (diluted 1:50, MO717; DAKO) in PB for 1 hour at room temperature, followed by incubation with a goat AMCA-conjugated anti-mouse antibody (diluted 1:2000, Alexa 350; Eugene, Leiden, The Netherlands) in PB for 1 hour at room temperature. Each detection step was followed by three washes for 10 minutes each in PBS containing $0.2 \%$ Triton X-100. After the last wash, slides were mounted in Vectashield (Vector Laboratories, Burlingame, California) mounting medium. Fifty randomly chosen cells from each slide were photographed using an Axioplan 2-fluorescence microscope (Imstar S.A., Paris, France). Subsequently, FISH was performed on the same slides using the probes described above, and the cells were photographed again. Comparison of immunofluorescence and FISH images allowed identification of cells with chromosome translocations within the populations of diverse cellular phenotype.

\section{Acknowledgement}

The authors thank Belinda Carleer, Lut Mekers, and Nancy Agten for their excellent technical assistance.

\section{References}

Batsakis JG (1980). Salivary gland neoplasia: An outcome of modified morphogenesis and cytodifferentiation. Oral Surg Oral Med Oral Pathol 49:229-232. 
Bullerdiek J, Raabe G, Bartnitzke S, Boschen C, and Schloot W (1987). Structural rearrangements of chromosome $\mathrm{Nr} 8$ involving 8q12: A primary event in pleomorphic ademona of the parotid gland. Genetica 72:85-92.

Dardick I, Rippstein P, Skimming L, Boivin M, Parks WR, and Dairkee SH (1987). Immunohistochemistry and ultrastructure of myoepithelium and modified myoepithelium of the ducts of human major salivary glands: Histogenetic implications for salivary gland tumors. Oral Surg Oral Med Oral Pathol 64:703715.

Dardick I, van Nostrand AW, and Phillips MJ (1982). Histogenesis of salivary gland pleomorphic adenoma (mixed tumor) with an evaluation of the role of the myoepithelial cell. Hum Pathol 13:62-75.

Dierlamm J, Wlodarska I, Michaux L, La Starza R, Zeller W, Mecucci C, and Van den Berghe H (1996). Successful use of the same slide for consecutive fluorescence in situ hybridization experiments. Genes Chromosomes Cancer 16:261-264.

Erlandson RA, Cardon-Cardo C, and Higgins PJ (1984). Histogenesis of benign pleomorphic adenoma (mixed tumor) of the major salivary glands. An ultrastructural and immunohistochemical study. Am J Surg Pathol 8:803-820.

Eversole LR (1971). Histogenic classification of salivary tumors. Arch Pathol 92:433-443.

Hay ED (1995). An overview of epithelio-mesenchymal transformation. Acta Anat 154:8-20.

Hockenbery DM, Oltvai ZN, Yin XM, Milliman CL, and Korsmeyer SJ (1993). Bcl-2 functions in an antioxidant pathway to prevent apoptosis. Cell 75:241-251.

Kas K, Voz ML, Hensen K, Meyen E, and Van de Ven WJM (1998). Transcriptional activation capacity of the novel PLAG family of zinc finger proteins. J Biol Chem 273:23026-23032.

Kas K, Voz ML, Roijer E, Astrom AK, Meyen E, Stenman G, and Van de Ven WJ (1997). Promoter swapping between the genes for a novel zinc finger protein and beta-catenin in pleomorphic adenomas with $\mathrm{t}(3 ; 8)(\mathrm{p} 21 ; \mathrm{q} 12)$ translocations. Nat Genet 15:170-174.

Lee PS, Sabbath-Solitare M, Redondo TC, and Ongcapin EH (2000). Molecular evidence that the stromal and epithelial cells in pleomorphic adenomas of salivary gland arise from the same origin: Clonal analysis using human androgen receptor gene (HUMARA) assay. Hum Pathol 31:498-503.
Neureiter D, Bohmer J, Kirchner T, and Aigner T (1999). Pleomorphic adenomas of the parotid express different mesenchymal phenotypes: Demonstration of matrix gene expression products characteristic of the fibroblastic and chondrocytic cell lineages. Histopathology 35:373-379.

Noguchi S, Aihara T, Yoshino K, Motomura K, Inaji H, Imaoka $\mathrm{S}$, and Koyama H (1996). Demonstration of monoclonal origin of human parotid gland pleomorphic adenoma. Cancer 77 : $431-435$.

Palmer RM, Lucas RB, Knight J, and Gusterson B (1985). Immunocytochemical identification of cell types in pleomorphic adenoma, with particular reference to myoepithelial cells. J Pathol 146:213-220.

Ron D and Dressler H (1992). PGSTag: A versatile bacterial expression plasmid for enzymatic labeling of recombinant proteins. Biotechniques 13:866-869.

Seifert G, Brocheriou C, Cardesa A, and Eveson JW (1990). WHO international histological classification of tumours. Tentative histological classification of salivary gland tumours. Pathol Res Pract 186:555-581.

Sunardhi-Widyaputra S and Van Damme B (1995). Immunohistochemical pattern of $\mathrm{Bcl}-2$ - and PTHrP-positive cells in primary, in recurrent and in carcinoma in pleomorphic adenomas. Pathol Res Pract 191:1186-1191.

van den Oord JJ, Sunardhi-Widyaputra S, Van Damme B, and De Ley M (1993). Monoclonal antibody to liver metallothionein: A novel marker for myoepithelial cells. Pathol Res Pract 189:1187-1190.

Voz ML, Agten NS, Van de Ven WJ, and Kas K (2000). PLAG1, the main translocation target in pleomorphic adenoma of the salivary glands, is a positive regulator of IGF-II. Cancer Res 60:106-113.

Voz ML, Astrom AK, Kas K, Mark J, Stenman G, and Van de Ven WJ (1998). The recurrent translocation $t(5 ; 8)(p 13 ; q 12)$ in pleomorphic adenomas results in upregulation of PLAG1 gene expression under control of the LIFR promoter. Oncogene 16:1409-1416. 